\title{
Prevalencia de nematodos gastrointestinales en alpacas y ovinos de dos cooperativas comunales de la región Pasco, Perú
}

\author{
Prevalence of gastrointestinal nematodes in alpacas and sheep from two \\ communal cooperatives in the Pasco region, Peru \\ Víctor Puicón ${ }^{1,3}$, Juan Chávez², Gustavo Gutiérrez², \\ Diana Sánchez ${ }^{1}$, Manuel More ${ }^{2}$, Daniel Zárate ${ }^{1}$
}

\section{Resumen}

\begin{abstract}
El objetivo del estudio fue determinar la prevalencia de nematodos gastrointestinales en alpacas Huacaya y ovinos Corriedale de plantel de dos cooperativas comunales de la región Pasco, Perú. Entre febrero de 2014 y abril de 2016 se colectaron 238 muestras fecales de alpacas y 319 de ovinos en San Pedro de Racco y 215 muestras fecales de ovinos y 178 de alpacas en Yurajhuanca. El contaje fecal de huevos de nematodos se determinó mediante la técnica de McMaster modificada y la identificación de los géneros parasitarios a través de la identificación morfométrica de larvas infectivas $\left(\mathrm{L}_{3}\right)$. Las prevalencias generales fueron de $65.2 \%$ (208/319) y $21.4 \%$ (51/238) para nematodos en ovinos y alpacas de la cooperativa San Pedro de Racco, y de 35.4\% (76/215) y 3.9\% (7/178) para nematodos en ovinos y alpacas de la cooperativa Yurajhuanca, respectivamente. Las especies halladas en ovinos y alpacas de ambas cooperativas fueron Oesophagostomum columbianum, Trichostrongylus columbiformis y Teladorsagia circumcincta.
\end{abstract}

Palabras clave: alpacas; ovinos; nematodos; Racco; Yurajhuanca; Pasco

\section{Abstract}

The aim of this study was to determine the prevalence of gastrointestinal nematodes in Huacaya alpacas and Corriedale sheep. Between February 2014 and April 2016, 238 faecal samples of alpacas and 319 of sheep were collected in San Pedro de Racco and 215 fecal samples of sheep and 178 samples of alpacas in Yurajhuanca. The faecal count of nematode eggs was determined by the modified McMaster technique and the identification

${ }^{1}$ Laboratorio de Parasitología, Departamento Académico de Nutrición, Facultad de Zootecnia, Universidad Nacional Agraria La Molina, Lima, Perú

2 Programa de Mejoramiento Genético Animal, Universidad Nacional Agraria La Molina, Lima, Perú

${ }^{3}$ E-mail: victor_puicon_1704@hotmail.com

Recibido: 9 de febrero de 2018

Aceptado para publicación: 12 de septiembre de 2018 
of the parasitic genus through the morphometric identification of infective larvae $\left(\mathrm{L}_{3}\right)$. The general prevalence was $65.2 \%$ (208/319) and 21.4\% (51/238) for nematodes in sheep and alpacas of the San Pedro de Racco cooperative, and of 35.4\% (76/215) and 3.9\% (7/178) for nematodes in sheep and alpacas of the Yurajhuanca cooperative, respectively. The species found in sheep and alpacas from both cooperatives were Oesophagostomum columbianum, Trichostrongylus columbiformis, and Teladorsagia circumcincta.

Key words: alpacas; sheep; nematodes; Racco; Yurajhuanca; Pasco

\section{INTRODUCCIÓN}

La producción ovina y de camélidos sudamericanos en el Perú representan en gran magnitud el sustento económico de un gran sector de la población rural (Moya y Torres, 2008); especialmente aquellas que habitan en zonas donde el desarrollo de la agricultura presenta limitantes, tanto por condiciones climáticas como altitudinales (INEI, 2012). La producción alpaquera en el Perú es una actividad ganadera nativa y de gran antigüedad que se conserva hasta a la actualidad, desarrollándose principalmente en sistemas extensivos y por las comunidades altoandinas (Moya y Torres, 2008).

Las condiciones naturales de crianza de ovinos y alpacas favorecen el desarrollo de diversas enfermedades parasitarias, ya que la limitada movilidad, la excesiva carga animal en las pasturas y el constante aumento en las exigencias productivas propicia el aumento de animales sensibles a las parasitosis (Waller, 2003). La crianza de estas especies en tales condiciones puede generar cuantiosas pérdidas económicas, las mismas que pueden incrementarse por efecto del cambio climático, al crearse un entorno nuevo más favorable para la aparición de los parásitos más patógenos, fenómeno que ya viene sucediendo en el país (Moya y Torres, 2008).

Este escenario exige enriquecer procesos tecnológicos y renovar conocimientos para poder generar estrategias de adaptación y prevención de enfermedades parasitarias, especialmente las provocadas por nematodos gastrointestinales. Una de las limitantes en el control de la nematodiasis es la resistencia que han venido adquiriendo frente a las drogas antihelmínticas (Kaplan, 2004; Gilleard, 2006; Pomroy, 2006). El objetivo del presente estudio fue determinar la prevalencia parasitaria en alpacas Huacaya y ovinos Corriedale de plantel, criados en pasturas naturales altoandinas, en dos granjas comunales de la región Pasco.

\section{Materiales Y MéTodos}

\section{Localidad del Estudio}

El presente estudio se realizó en las cooperativas de San Pedro de Racco y la Unidad de producción Ayaracra de la Cooperativa Yurajhuanca, localizadas en la provincia de Daniel Alcides Carrión, Región Pasco, Perú. La zona presenta altitudes entre 4375 y 4350 msnm y un rango de temperatura anual entre 0 y $9{ }^{\circ} \mathrm{C}$.

\section{Población de Animales}

Ambas cooperativas tienen alrededor de cuatro mil alpacas Huacaya y siete mil ovinos Corriedale, divididas en majada y plantel de acuerdo con sus parámetros productivos. Se trabajó con los animales de plantel por poseer registros genealógicos y productivos. Los animales en estudio correspondieron a adultos, jóvenes y crías de las temporadas secas y lluviosas de 2014 y 2015 ; mientras que en las alpacas se incluyó, además, la época lluviosa de 2016. Se tomaron muestras a to- 
Cuadro 1. Número total de ovinos (2014 y 2015) y alpacas (2014-2016) muestreados para la evaluación de la prevalencia parasitaria

\begin{tabular}{lcc}
\hline & Ovinos & Alpacas \\
\hline $\begin{array}{l}\text { San Pedro de } \\
\text { Racco }\end{array}$ & 319 & 238 \\
Yurajhuanca & 215 & 178 \\
\hline
\end{tabular}

dos los animales que presentaron registros productivos (Cuadro 1); sin embargo, los intervalos tomados en cada año para ambas cooperativas, el número de animales muestreados y la paridad de los mismos animales variaron en cada campaña de muestreo ante la limitada disponibilidad de la cooperativa y el diferente flujo de animales de saca.

En la evaluación de las muestras se consideró el grupo etario (alpacas: crías [desde el nacimiento hasta los 16 meses], jóvenes [desde los 16 meses hasta los 24 meses] y adultos [desde los 24 hasta los 60 meses]; ovinos: cría [desde el nacimiento hasta los 12 meses], borrega [desde los 12 hasta los 18 meses], oveja [a partir de los 24 meses]).

\section{Análisis Parasitológico}

Las fechas de las tomas de muestras de heces fue hecha en consideración al calendario de dosificación que manejaban las cooperativas. Así, en el caso de ovinos en San Pedro de Racco se tomaron las muestras un mes antes y un mes después de las dosificaciones programadas, las cuales fueron realizadas con antiparasitarios como albendazol, praziquantel e ivermectina. En el caso de Yurajhuanca se dosifica tres veces al año, siendo en los meses de abril (con triclalbendazol), agosto (con albendazol) y diciembre (con rafoxanide + albendazol), ya que las principales parasitosis registradas son la nematodiasis neumogastroentérica y la distomatosis hepática. En este caso, la toma de muestras se hizo antes del primer tratamiento antihelmíntico en abril, luego de dos meses del segundo tratamiento (setiembre) y antes del último tratamiento realizado en octubre y luego de un mes (noviembre). En el caso de alpacas, las dosificaciones se realizaron cada cuatro meses (enero, mayo y agosto o setiembre con albendazol al $12.5 \%$, en ambas cooperativas De esta forma, la toma de muestras fue previa a las desparasitaciones.

Las heces fueron recolectadas directamente del recto y colocadas en bolsas de polietileno con la identificación del animal. Las muestras se transportaron en cajas isotérmicas de poliestireno expandido con geles refrigerantes al Laboratorio de Parasitología de la Facultad de Zootecnia de la Universidad Nacional Agraria La Molina (FZ-UNALM), Lima. El tiempo transcurrido entre la colección de las muestras y su análisis fue entre 1 y 2 días.

Las muestras fecales se analizaron mediante la técnica McMaster modificado (Ueno y Gonçalves, 1998) para obtener el número de huevos por gramo de heces (HPGH) de nematodos tipo estrongílido (HTS) y de otras especies (Nematodirus sp, Lamanema sp y Trichuris sp). Las muestras positivas a HTS fueron sometidas a cultivo de larvas en pooles de adultos y crías. Las larvas infectivas (L3) fueron recuperadas mediante el método de Baermann (Ueno y Gonçalves, 1998).

Los géneros de las larvas infectivas fueron determinados mediante el reconocimiento de sus características morfológicas y biométricas utilizando el software Leica Application Suite v. 4.0 (van Wyk y Mayhew, 2013), que se basa en medidas como el total de la longitud de la larva, medición de la longitud de la cola y forma de la cabeza, así como el contaje del número de células gastrointestinales. 

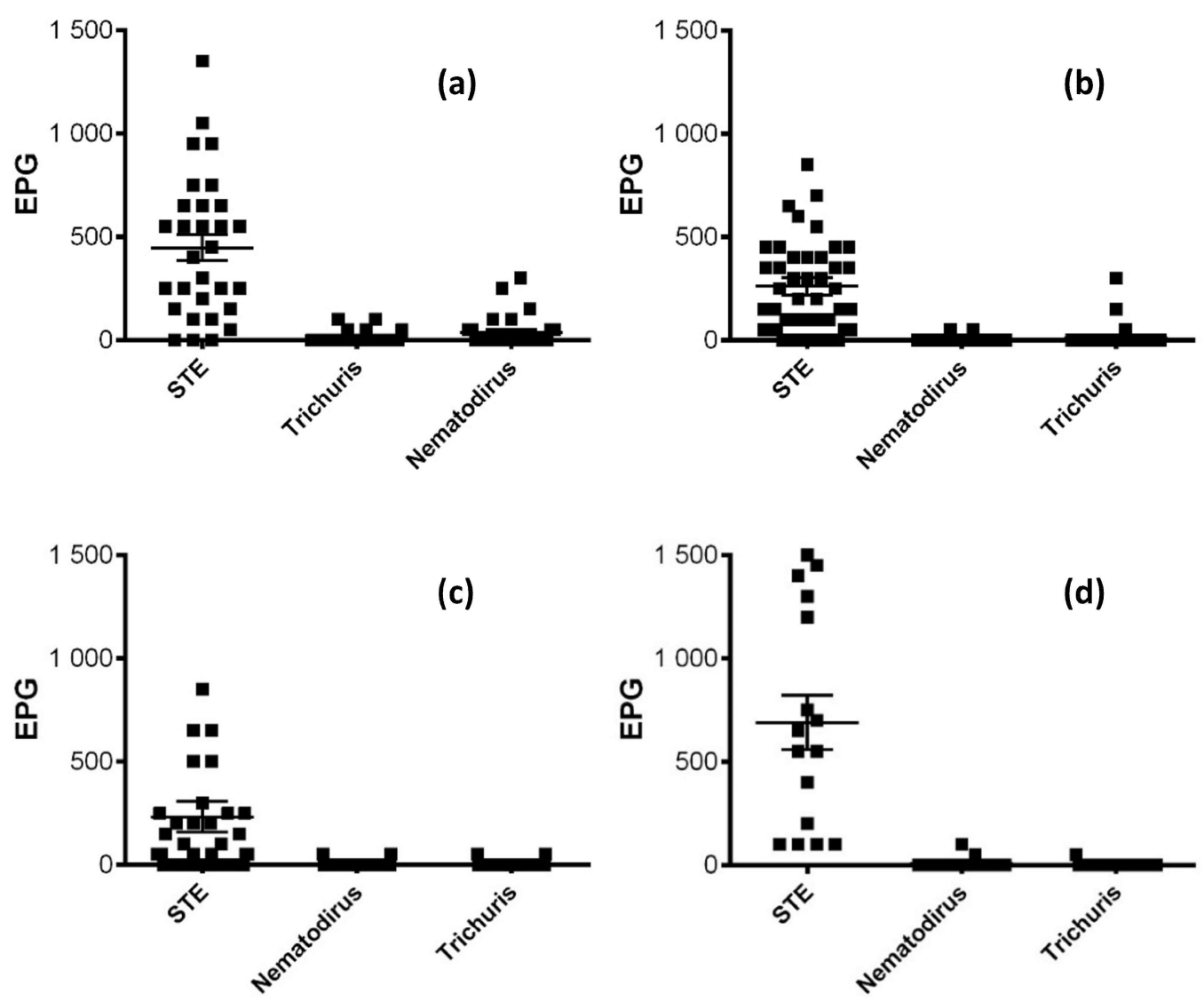

Figura 1. Dinámica parasitaria en el rebaño de ovinos de plantel de la cooperativa de San Pedro de Racco en 2014. (a) febrero, (b) agosto, (c) octubre, (d) noviembre. STE: huevo tipo estrongílido; EPG: huevos por gramo de heces

\section{Análisis Estadístico}

Se calculó la prevalencia de infección parasitaria mediante la determinación del número de muestras fecales positivas. La dinámica parasitaria fue representada con gráficos mediante el programa Graphpad Prism 6 (Software Inc, EEUU).

\section{Resultados y Discusión}

\section{Plantel de Ovinos}

Cooperativa comunal de San Pedro de Racco

La prevalencia de parasitismo gastrointestinal en ovinos de San Pedro de Racco- para los dos años del estudio fue de $65.2 \%$ (208/319) y en Yurajhuanca de 35.4\% (76/215) en un año de estudio.

En 2014, en época de lluvias (febrero) se hallaron cargas HTS de leves a moderadas en crías (525 hpg) y adultos (480 hpg). En época seca (agosto), la crías posdestete obtuvieron mayoritariamente cargas elevadas alcanzando niveles de hasta $2850 \mathrm{hpg}$ (media: $1322 \mathrm{hpg}$ ). En octubre predominaron las cargas leves, seguido de cargas moderadas (445 hpg en crías, 207 hpg en adultos), mientras que en noviembre predominaron las cargas moderadas y elevadas en todas las categorías (Figura 1). Trichuris sp fue hallado con mayor frecuencia, llegando a alcanzar cargas moderadas. Nematodirus sp y Trichuris sp fueron hallados predominantemente en crías. 

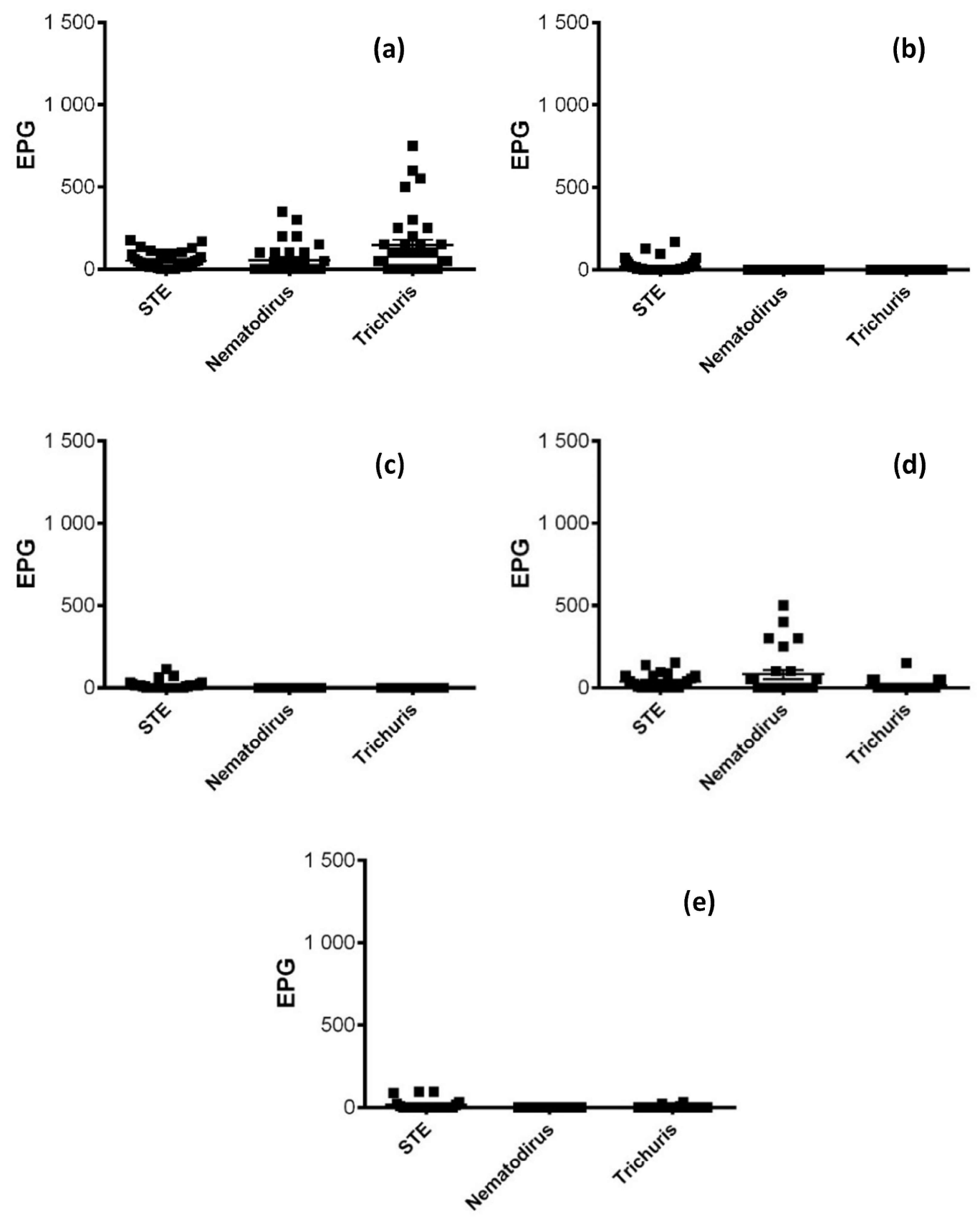

Figura 2. Dinámica parasitaria en el rebaño de ovinos de plantel de la cooperativa de San Pedro de Racco en 2015. (a) abril, (b) julio, (c) septiembre, (d) octubre, (e) noviembre. STE: huevo tipo estrongílido; EPG: huevos por gramo de heces

Las cargas parasitarias moderadas a elevadas de octubre y noviembre coinciden con la temporada posparto y lactación de las hembras reproductoras, donde usualmente se observan elevados recuentos de hpg entre las 2 y 4 semanas posparto (Goldberg et al.,
2012) y entre 6 y 8 semanas posparto (Nari et al., 1977). Las hembras posparto son las más afectadas en situaciones de estrés, ya que se genera una ruptura de la inmunidad y, por consecuente, las larvas arrestadas por hipobiosis reinician su desarrollo (Abbott $e t$ 
al., 2012). Se considera que la contaminación de los pastos con huevos de parásitos gastrointestinales corresponde al $75 \%$ a las borregas y el $25 \%$ a las crías posdestete (Leguía y Casas, 1999).

En 2015, las cargas HTS se caracterizaron por ser leves, tanto en adultos como en crías, sin sobrepasar el promedio de $50 \mathrm{hpg}$. En el primer y penúltimo muestreo (abril y octubre) (Figura 2a,d) se observa un mayor número de Trichuris sp y Nematodirus sp, especialmente en crías. Lamentablemente, una proporción de ovejas gestantes no estuvieron disponibles para el muestreo.

En el cultivo de larvas de ovinos adultos de agosto de 2014 se obtuvieron las siguientes frecuencias: Oesophagostomum columbianum 41\%, Trichostrongylus columbiformis $25 \%$, Chabertia ovina $13 \%$, Ostertagia ovina $12 \%$ y Teladorsagia circumcincta $9 \%$. En el muestreo de octubre en crías en 2014 se obtuvo Chabertia ovina 48\%, Trichostrongylus columbiformis $24 \%$, Oesophagostomum columbianum $24 \%$ y Teladorsagia circumcincta $4 \%$. Los porcentajes de larvas infectivas se mantuvieron en las mismas especies en ambos muestreos; sin embargo, se observó una disminución de la frecuencia de larvas de Oesophagostomum columbianum y un aumento de Chabertia ovina a fines de la época seca, aunque sin una estacionalidad marcada en ambas especies.

En tanto, en 2015 se obtuvo similitud en las frecuencias de las crías de ovinos. En el muestreo de abril se obtuvieron las siguientes frecuencias: Chabertia ovina 54\%, Trichostrongylus colubriformis $22 \%$, Oesophagostomum columbianum 20\% y Teladorsagia circumcincta 4\%. En el muestreo de julio se tuvo Chabertia ovina $46 \%$, Oesophagostomum columbianum $24 \%$, Trichostrongylus colubriformis $20 \%$ y Teladorsagia circumcincta $10 \%$.
El principal nematodo en ovinos y caprinos a nivel mundial es Haemonchus contortus (Wanyangu et al., 1997; Mandonnet et al., 2001; Goldberg et al., 2012), seguido de Trichostrongylus spp (Nari et al., 1977; Castells, 2009), y con variaciones de acuerdo a la época del año de Oesophagostomum columbianum, Cooperia curticei, Trichostrongylus vitrinus, Trichostrongylus colubriformis y Nematodirus fillicolis (Sykes, 1978). A diferencia del presente estudio, Haemonchus contortus no fue hallado, posiblemente debido las condiciones poco favorables para su desarrollo (clima frío, gran altitud y poca humedad de la sierra central del Perú) (Chaudary et al., 2008).

\section{Cooperativa comunal Yurajhuanca}

En los muestreos de 2015 se halló $57.0 \%$ (57/100) de animales parasitados en abril y $17.0 \%$ (18/106) en julio. En el muestreo de setiembre solo se colectaron nueve muestras, donde un animal tuvo una carga leve. Todos los animales muestreados en abril fueron adultos, con un promedio de $71 \mathrm{hpg}$. En julio se muestrearon a 57 adultas y 49 crías, donde tanto las adultas (9/57) como las crías (9/49) tuvieron cargas leves con un promedio de 14.2 hpg y $17.77 \mathrm{hpg}$, respectivamente, sin que se hallasen huevos diferentes a HTS.

Las especies halladas en el coprocultivo realizado en los animales adultos fueron de Chabertia ovina (72.5\%), Trichostrongylus colubriformis $(20 \%)$ y Teladorsagia circumcincta $(7.5 \%)$, resultados que coinciden con la cooperativa comunal de San Pedro de Racco.

\section{Plantel de Alpacas}

Se determinó una prevalencia total de 21.4\% (51/238) en San Pedro de Racco y de $3.9 \%$ (7/178) en Yurajhuanca para los años 2014,2015 y 2016, lo cual es inferior a repor- 
tes de $68.4 \%$ en Cusco y de 33.8-85.0\% en distritos de Puno (Melo, 1997; CEDER, 2009; Contreras et al., 2014).

\section{Cooperativa comunal de San Pedro de} Racco

En la época seca de 2014 (agosto) se hallaron cargas parasitarias leves al analizarse un total de 67 alpacas. En adultos y crías se hallaron cargas promedio de $50 \mathrm{hpg}$. En animales jóvenes además se hallaron los géneros Nematodirus y Trichuris, hallándose el segundo con cargas de hasta $250 \mathrm{hpg}$. Para este muestreo se realizó un coprocultivo de las alpacas adultas hallándose las especies Trichostrongylus colubriformis (66\%), Teladorsagia circumcincta (32\%) y Oesophagostomum columbianum (2\%).

En la época de lluvias (enero de 2015) se hallaron cargas de hpg con niveles nulos a leves y los nematodos de los géneros Nematodirus y Trichuris no tuvieron cargas mayores de $50 \mathrm{hpg}$. En la época seca (octubre de 2015) hubo predominancia de cargas nulas, tanto en adultos como en crías, donde los pocos animales con recuentos leves no tuvieron cargas mayores de $150 \mathrm{hpg}$.

En 2016 se realizó un seguimiento de las crías posdestete tanto en los meses de enero (época de lluvias) y abril (época seca). En el primer muestreo, las crías fueron positivas con cargas leves con promedio de 11.48 hpg. Así mismo, se hallaron parásitos de los géneros Nematodirus y Trichuris con cargas leves. En abril, los animales adultos presentaron cargas leves para Nematodirus sp, Trichuris sp y Lamanema sp, con un promedio de $18.78 \mathrm{hpg}$ para huevos HTS.

Las diferencias entre las especies parasitarias halladas en este estudio con otros reportes se deben principalmente a las diferencias geográficas, donde por ejemplo Haemonchus contortus es el nematodo de mayor perjuicio a nivel mundial en ovinos y camélidos (Sarre et al., 2012; Jabbar et al., 2013), el cual predomina en climas tropicales y subtropicales, en tanto que nematodos como Trichostrongylus sp y Nematodirus sp pueden convivir en climas fríos (Barriga, 2002).

\section{Cooperativa comunal Yurajhuanca}

Las condiciones climáticas en Yurajhuanca tienden a ser muy marcadas, caracterizándose por la alta precipitación pluvial y granizo, lo que genera un clima adverso, que genera un estado de quiescencia de las larvas $\mathrm{L} 4$, las cuales pueden permanecer durante meses sin desarrollarse en la mucosa estomacal e intestinal (Leguía y Casas, 1999); sin embargo, en los primeros muestreos se registraron cargas moderadas de Nematodirus sp y Trichuris sp, cuyas larvas infectivas se desarrollan dentro de los huevos, constituyendo una protección al medio ambiente adverso (Leguía y Casas, 1999).

No se pudo concluir que las alpacas de menor edad sean las que estén mayormente infectadas. Tampoco se pudo observar cargas moderadas ni altas en las tres categorías de animales, lo que coincide con estudios realizados en Cusco (Pérez et al., 2014), Puno (Contreras et al., 2014) y Pasco (Masson et al., 2016).

\section{Conclusiones}

- La prevalencia general de parasitismo gastrointestinal por nematodos en ovinos fue $65.2 \%$ (208/319) y $35.4 \%$ (76/215), en las cooperativas comunales de San Pedro de Racco y Yurajhuanca, respectivamente.

- La prevalencia general de parasitismo gastrointestinal por nematodos en alpacas fue de $21.4 \%$ (51/238) y de $3.9 \%$ (7/178), en las cooperativas comunales de San Pedro de Racco y Yurajhuanca, respectivamente.

- Se encontraron Oesophagostomum columbianum, Trichostrongylus columbiformis y Teladorsagia circumcincta en ovinos y alpacas, además de 
Ostertagia ovina y Chabertia ovina en ovinos.

- Parásitos de los géneros Nematodirus sp y Trichuris sp fueron hallados en ambas especies y en ambas localidades.

\section{Agradecimientos}

El presente estudio fue financiado por el Consejo Nacional de Ciencia y Tecnología e Innovación tecnológica (CONCYTEC) y el programa de cooperación interuniversitaria VLIR UOS - UNALM, y fue realizado gracias al apoyo de la Cooperativa Comunal San Pedro de Racco y la Unidad de Producción Ayaracra de la Cooperativa Comunal Yurajhuanca.

\section{Literatura Citada}

1. Abbott KA, Taylor M, Stubbings LA. 2012. Sustainable worm control strategies for sheep. A technical manual for veterinary surgeons and advisers. $4^{\text {th }}$ ed. UK: Scops. 55 p.

2. Barriga O. 2002. Las enfermedades parasitarias de los mamíferos dómesticos en America Latina. Santiago: Germinal. $334 \mathrm{p}$.

3. Castells D. 2009. Evaluación de resistencia genética de ovinos Corriedale a los nematodos gastrointestinales en Uruguay: heredabilidad y correlaciones genéticas entre el recuento de huevos de nematodos y características productivas. Tesis de Maestría. Montevideo, Uruguay; Universidad de la República. $54 \mathrm{p}$.

4. [CEDER] Centro de Estudios para el Desarrollo Regional. 2009. Desarrollo de las capacidades productivas y comerciales de los pequeños criadores de alpacas de los distritos de Mañazos y Cabanillas. Puno, Perú: CEDER. 28 p.

5. Chaudary F, Qayyum M, Miller JE. 2008. Development and survival of Haemonchus contortus infective larvae derived from sheep faeces under sub- tropical conditions in the Potohar region of Pakistan. Trop Anim Health Prod 40: 85-92.

6. Contreras $N$, Chávez A, Pinedo $R$, Leyva V, Suárez F. 2014. Helmintiasis en alpacas (Vicugna pacos) de dos comunidades de Macusani, Puno, durante la época seca. Rev Inv Vet Perú 25: 268275. doi: 10.15381/rivep.v25i2.8499

7. Gilleard JS. 2006. Understanding anthelmintic resistance: the need for genomics and genetics. Int J Parasitol 36: 1227-1239. doi: 10.1016/j.ijpara.2006.06.010

8. Goldberg V, Ciappesoni G, Aguilar I. 2012. Genetic parameters for nematode resistance in periparturient ewes and post-weaning lambs in Uruguayan Merino sheep. Livest Sci 147: 181-187. doi: 10.1016/j.livsci.2012.05.003

9. [INEI] Instituto Nacional de Estadística e Informática. 2012. IV Censo Nacional Agropecuario 2012. [Internet]. Disponible en: http://proyectos.inei.gob.pe/web/DocumentosPublicos/ ResultadosFinalesIVCENAGRO.pdf

10. Jabbar A, Angus JD, Campbell JA, Gasser RB. 2013. First report of anthelmintic resistance in Haemonchus contortus in alpacas in Australia. Parasite Vector 6: 243. doi: 10.1186/17563305-6-243

11. Kaplan RM. 2004. Drug resistance in nematodes of veterinary importance: a status report. Trends Parasitol 20: 477481. doi: 10.1016/j.pt.2004.08.001

12. Leguía G Casas E. 1999. Enfermedades parasitarias y atlas parasitológico de camélidos sudamericanos. Lima, Perú: Editorial De Mar. $190 \mathrm{p}$.

13. Mandonnet N, Aumont G, Fleury R, Arquet H, Varo L, Gruner J, Bouix J, et al. 2001. Assessment of genetic variability of resistance to gastrointestinal nematode parasites in Creole goats in the humid tropics. J Anim Sci 79: 1706-1712. doi: $10.2527 / 2001.7971706 x$

14. Masson M, Gutiérrez G, Puicón V, Zárate D. 2016. Helmintiasis y eimeriosis gastrointestinal en alpacas 
criadas al pastoreo en dos granjas comunales de la región Pasco, Perú, y su relación con el peso y condición corporal. Rev Inv Vet Perú 27: 805-812. doi: 10.15381/rivep.v27i4.12566

15. Melo A. 1997. Sistemas de control y manejo sanitario de las alpacas y llamas en la región andina del sur peruano. Rev FMVZ-UNA 1: 54-59.

16. Moya E, Torres J. 2008. Familias alpaqueras enfrentando al cambio climático. Lima. 110 p. [Internet]. Disponible en: https://www.mimp.gob.pe/ webs $/ \mathrm{mimp} / \mathrm{sispod} / \mathrm{pdf} / 186 . \mathrm{pdf}$

17. Nari A, Cardozo H, Berdié J, Canábez F, Bawden R. 1977. Dinámica de población para nematodos gastrointestinales de ovinos en el Uruguay. Veterinaria 14: 11-24.

18. Pérez H, Chávez A, Pinedo R, Leyva V. 2014. Helmintiasis y eimeriasis en alpacas de dos comunidades de Cusco, Perú. Rev Inv Vet Perú 25: 245-253. doi: 10.15381/rivep.v25i2.8497

19. Pomroy WE. 2006. Anthelmintic resistance in New Zealand: a perspective on recent findings and options for the future. New Z Vet J 54: 265-270. doi: 10.1080/00480169.2006.36709
20. Sarre C, Claerebout E, Vercruysse $J$, Levecke B, Geldhof P, Pardon B, Alvinerie M, et al. 2012. Doramectin resistance in Haemonchuscontortus on an alpaca farm in Belgium. Vet Parasitol 185: 346-351. doi: 10.1016/ j.vetpar.-2011.10.003

21. Sykes AR. 1978. The effect of subcli-nical parasitism in sheep. Vet Rec 102: 32-34.

22. Ueno H, Goncalves PC. 1998. Manual para diagnostico das helmintoses de ruminantes. Tokio, Japón: JAICA. $116 \mathrm{p}$.

23. van Wyk JA, Mayhew E. 2013. Morphological identification of parasitic nematode infective larvae of small ruminants and cattle: A practical lab guide. Onderstepoort J Vet 80: a539. doi: 10.4102/ojvr.v80i1.539

24. Waller PJ. 2003. Global perspectives on nematode parasite control in ruminant livestock: the need to adopt alternatives to chemotherapy, with emphasis on biological control. Anim Health Res Rev 4:35-43. doi: 10.1079/AHRR200350

25. Wanyangu JM, Mugambi RK, Bain JL, Duncan M, Murray MJ, Stear B. 1997. Response to artificial and subsequent natural infection with Haemonchus contortus in Red Maasai and Dower ewes. Vet Parasitol 6: 275282. doi: 10.1016/S0304-4017(96)01129-6 